\title{
Discriminative control and response maintenance by a brief aversive stimulus in a fixed-interval schedule
}

\author{
BROCK KILBOURNE and ROBERT A. FOX \\ San Jose State University, San Jose, California 95192
}

\begin{abstract}
The discriminative and suppressive properties of a brief aversive light intruded into a fixed-interval schedule were investigated using 24 albino rats. The aversive light was intruded into the early, middle, or terminal $6 \mathrm{sec}$ of a FI 60-sec food-reinforcement schedule for three groups of eight rats each. After 5 days of training, rats which initially had the light paired with reinforcement received the light in early and initial FI periods on 2 transfer test days. When the light was paired with reinforcement, response suppression during the light was minimized. In transfer tests, after the light was initially paired with reinforcement two effects existed: (1) Response suppression was decreased during the light, and (2) response facilitation occurred immediately following the light. Pervasive control by the FI schedule was reflected in a return to the baseline response rate following response facilitation. Reduced suppression during the light and response facilitation following light offset were ascribed to discriminative properties resulting from the predictive temporal relationship between the light and reinforcement opportunity.
\end{abstract}

Single stimuli intruded into fixed-interval (FI) schedules acquire response-maintaining properties when occurring late in the interval (near reinforcement availability) and suppressive properties when occurring in central portions of the interval. This basic effect has been demonstrated with both neutral (Farmer \& Schoenfeld, 1966) and aversive stimuli (Snapper, Kadden, Shimoff, \& Schoenfeld, 1975). However, when Farmer and Schoenfeld presented a neutral light twice within each FI segment, pigeons tended to respond during both presentations of the light and to pause when the light was absent if the second light was contiguous with reinforcement. With this procedure, the response-maintaining properties of the intruded stimulus were apparently dependent upon pairing the light with reinforcement.

Using this double-stimulus method, Farmer and Shoenfeld (1966) demonstrated a gradient of discriminative control along a temporal continuum, with response strength systematically decreasing as the stimulus became farther removed from reinforcement. Since the stimulus maintained control over responding at long temporal intervals from reinforcement, Farmer and Schoenfeld concluded that it functioned as a discriminative stimulus.

This research was submitted by Brock Kilbourne in partial fulfillment of the requirements for the MA degree. This paper is an expanded version of a paper delivered at the Western Psychological Association meetings in Los Angeles, April 1976. The authors wish to thank Ronald Rabedeau, who sponsors this paper, for his helpful comments during the design of this research and for his editorial assistance in the preparation of this paper. Requests for reprints should be sent to Robert A. Fox, Department of Psychology, San Jose State University, San Jose, California 95192.
Using a single aversive stimulus paired with reinforcement, Snapper et al. (1975) found suppression of response rate prior to the stimulus and no change in response rate during the stimulus paired with reinforcement. They argued that a discriminative stimulus interpretation of their data does not accord with current usage of the term because behavior in their study was modified in the absence of the stimulus, but not in its presence. These authors suggested that delay of punishment may have produced their results.

The purpose of the present experiment was to study further the discriminative and suppressive properties of an aversive stimulus paired with reinforcement in a FI schedule. A brief aversive light was intruded into one half of the FI segments of each experimental session. The behavioral effects of this light were measured by comparing responding during the light to responding in the same portion of the fixed interval when the light was absent. Following initial testing with the aversive light presented immediately preceding reinforcement availability, the effect of the light was tested by shifting it to the initial and middle segments of the FI. The performance of a transfer group was then compared to that of other groups always having the light intruded in the initial and middle FI segments.

\section{METHOD}

\footnotetext{
Subjects

Twenty-four male albino rats, approximately 150 days old, were maintained at $80 \%$ of free-feeding body weight throughout the experiment. Eight rats were randomly assigned to each of the three treatment conditions. One rat died and one failed to develop a scallop response pattern; both were replaced by the same type of rats of approximately the same age.
} 


\section{Apparatus}

Two Scientific Prototype (Model SP 300) experimental chambers, modified by raising the response lever to a location $8.26 \mathrm{~cm}$ above the apparatus floor, were used. A force of about $15 \mathrm{~g}$ was required for lever operation in each chamber. Ambient illumination of $2 \mathrm{fc}(21.5 \mathrm{lux})$ was provided by the chamber lever light (a GE 1829 lamp). The aversive light was a 100-W bulb, centered $37.5 \mathrm{~cm}$ above each experimental chamber, that increased the illuminance to $400 \mathrm{fc}(4,305.6 \mathrm{lux})$ when illuminated. Each experimental chamber was housed inside a larger sound-attenuating chamber, and a $98-\mathrm{dB}$ re $20 \mathrm{~N} / \mathrm{m}^{2}$ background noise was used to mask extraneous sounds. Programming was accomplished with solid state logic equipment.

\section{Procedure}

After falling to $80 \%$ of free-feeding body weight, each rat was randomly assigned to one of the two experimental chambers and hand shaped. A continuous schedule of reinforcement was used to establish leverpressing for $45-\mathrm{mg}$ Noyes food pellets. After shaping, each rat was placed on a FI 30-sec schedule for 2 days and earned 60 reinforcements each day. This was followed by a FI 60-sec schedule for an average of 8 days (range of 7 to 10) to establish FI performance. The criterion for baseline was 5 consecutive days of consistent FI scalloping determined by visual inspection of cumulative records.

Each experimental session contained 60 FI segments and was terminated after the 60th food pellet was earned. During Days 1-5 a 6-sec aversive light was presented at one of three different temporal locations during the FI 60 . The aversive light occupied the 6-sec interval from 12 to $18 \mathrm{sec}$ (early segment), 30 to $36 \mathrm{sec}$ (middle segment), and 54 to $60 \mathrm{sec}$ for Groups 1, 2 , and 3 , respectively. In each group the aversive light was intruded into one half of the $60 \mathrm{FI}$ segments composing an experimental session. The 30 intrusions were randomly distributed among the $60 \mathrm{FI}$ segments of each session.

On Days 6 and 7 the same procedure was continued for Groups 1 and 2. The eight animals in Group 3 were randomly assigned to two transfer subgroups to test effects of the 5 preceding treatment days. For one half of the transfer animals, the light occupied the early segment on Day 6 and the middle segment on Day 7; for the other half, the early and middle segment order was reversed on Days 6 and 7.

\section{RESULTS}

Suppressive effects of the aversive light were determined by contrasting responding during the 6-sec period of the FI when the light was present to responding in the same 6-sec period when the light was absent. The amount of responding when the light was absent served as a baseline response measure. Response frequency during the $150 \mathrm{FI}$ sequences with a light (30 in each of five sessions) was divided by the sum of response frequencies in the same 150 sequences and the 150 sequences when no light was present. With this measure, a ratio of .50 indicates no suppression is occurring, with ratios approaching zero reflecting increasing suppression.

Response suppression in Days 1.5 was assessed by using the response baseline determined in those five sessions. Suppression ratio means were compared using Duncan's multiple range test. Group 2 (mean $=.15$ ) suppressed more than Group $3($ mean $=.43)(p<.05)$, but Group 1 (mean $=.22$ ) and Group 3 did not differ.

As measured by mean total responses, Group 1 animals responded twice as much in the 6-sec target interval during the five FI performance establishing sessions (.52) as during Days 1-5 (.28). Both Group 2 (.82 and .70) and Group 3 (3.00 and 3.03) reflected only a small difference in responding during the same two periods. It appears that the aversiveness of the intruded light produced generalized suppression, which resulted in a "floor effect" that reduced the measured suppression. In Group 1 the early segment responding fell so low, even with no light present, that it became difficult to detect response suppression. However, when the suppression ratio data of Groups 1 and 3 are computed using responding during the five FI performance establishing sessions as a baseline, there is significantly more suppression in Group 1 than in Group 3 $[\mathrm{t}(14)=7.25, \mathrm{p}<.001]$.

Both suppression ratios and response rate were examined on the two transfer test sessions (Days 6-7). Using Student's $t$ for independent samples, comparison of suppression ratios for transferred animals (Group 3) with those continuing in the same conditions (Groups 1-2) indicated decreased suppression during the light (see upper panels of Figure 1). Animals transferred to the early segment on Day 6 were transferred to the middle segment on Day 7 , and vice versa. Decreased suppression was present for both probe segments on Day 6 and for the middle probe segment on Day 7 [all
EARLY SEGMENT
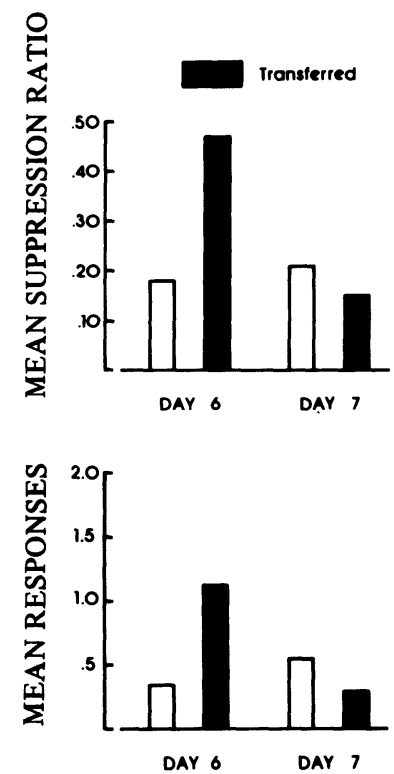

MIDDLE SEGMENT
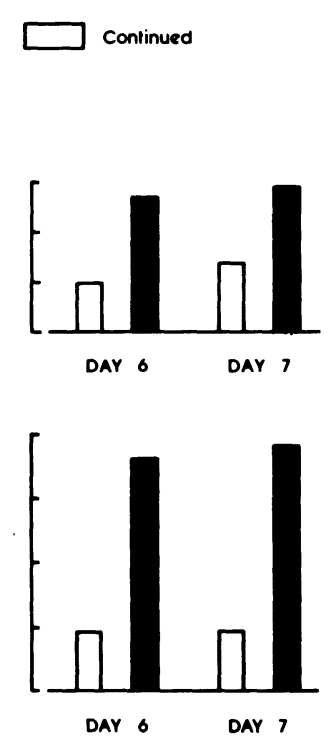

Figure 1. Mean suppression ratios during light presentations (upper panels) and mean total responses in the 6-sec interval following light presentation (lower panels) for transferred animals (Group 3) and animals trained with the light occurring only in early or middle segments (Groups 1 and 2). Transferred animals tested in the early segment on Day 6 were tested in the middle segment on Day 7, and vice versa. 


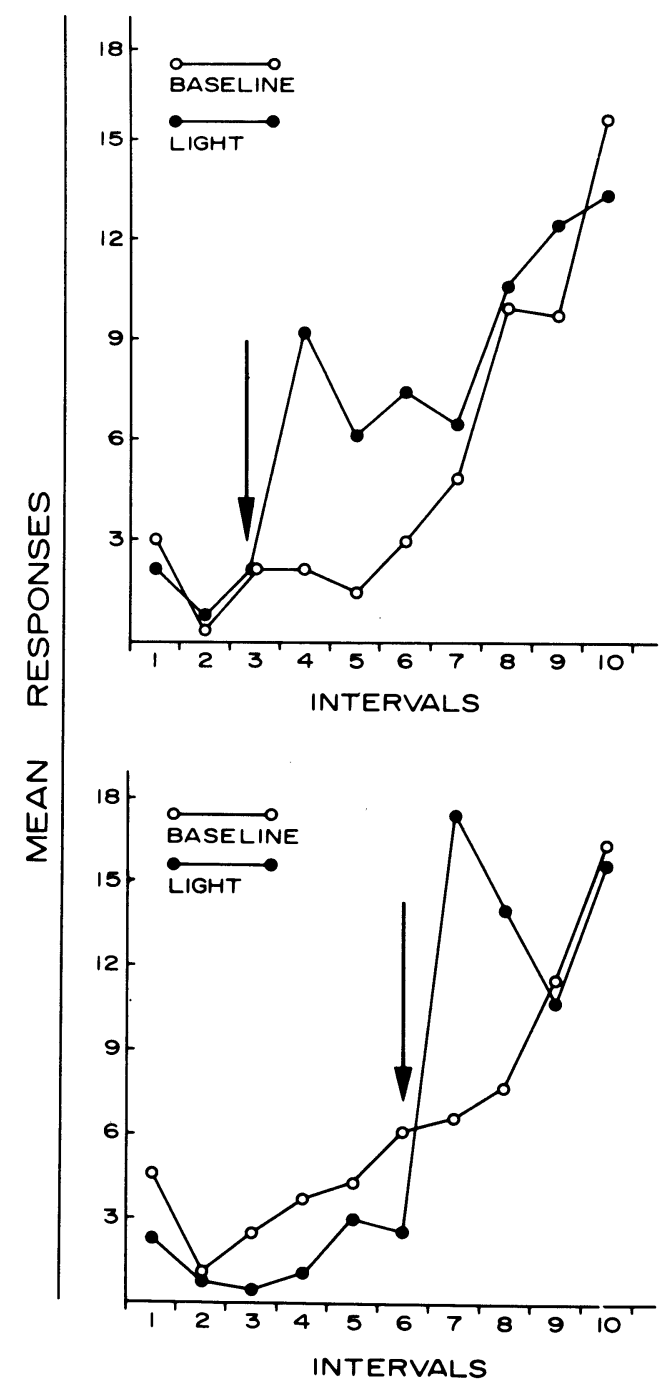

Figure 2. Mean total responses by transferred animals in each 6-sec interval of the FI cycle during transfer session baseline and light sequences for the first five light presentations on the first transfer test day (Day 6). Arrows indicate the interval containing the light.

$\operatorname{ts}(10) \geqslant 3.00, \mathrm{p}<.02]$ but not for the early probe segment on Day $7[\mathrm{t}(10)=.35, \mathrm{p}>.20]$. Response facilitation for Group 3 following the light was evident in the comparison of mean responses for postlight intervals (see lower panels of Figure 1). As with suppression during the light, facilitation in the postlight interval occurred for both segments on Day 6 but for only the middle segment on Day 7 [all ts $(10) \geqslant 3.09, p<.02$ ] No facilitation was evident in the early segment on Day $7[\mathrm{t}(10)=.75, \mathrm{p}<.20]$. This postlight facilitation effect was followed by a return to baseline response rate, indicating control by the FI schedule. The combined effects of suppression during the light, facilitation following the light, and the FI schedule was to produce a "double scallop" response pattern. This result is shown for the first five transfer light presentations of Group 3 in Figure 2.

\section{DISCUSSION}

When the aversive light occurred immediately prior to reinforcement, response suppression during the light was minimized. After the light had initially occurred just before reinforcement availability, response suppression was reduced in transfer tests. Additionally, in transfer, response facilitation occurred after light offset and was followed by a return to local baseline rates within the same FI sequence. The light always occurred during a period of reinforcement unavailability and might be expected to produce response suppression as an $S \Delta$ in addition to being averisive. The minimal response suppression observed when the light immediately preceded reinforcement indicates it was an ineffective $S^{\Delta}$ for Group 3 animals. When the light immediately precedes reinforcement availability, it apparently serves to predict reinforcement and acquires discriminative properties. These discriminative properties counteract the aversive characteristics of the stimulus, resulting in the reduced response suppression found in Group 3. Similar effects have been demonstrated using a shock with FI scheduling by Holz and Azrin (1962) and are also in agreement with the demonstration that the temporal relationship of a brief stimulus to reinforcement opportunity is an important determiner of stimulus control in intermittent schedules (Cohen \& Stubbs, 1976). Response facilitation following light offset in transfer may be taken to reflect the strength of the light's discriminative property in the absence of suppression.

In the present study the aversive stimulus was intruded into $50 \%$ of the reinforcement intervals rather than into each interval, as in prior studies (Farmer \& Schoenfeld, 1966; Holz \& Azrin, 1962; Snapper et al., 1975). With these conditions, the FI schedule continued to maintain control and the FI scallop was maintained. During transfer, the discriminative property continued to counteract response suppression during the light. When reinforcement did not occur following the light, there was a recovery of the baseline response rate, reflecting the strong control exerted by the temporal cue.

Snapper et al (1975) suggested that the differential suppression found with this procedure could be attributed to a delay of punishment effect. The time between reinforcement and aversive stimulus onset in the succeeding FI is interpreted as a punishment delay interval. With this interpretation, the present experiment uses a $50 \%$ partial punishment procedure with delays of 12 , 30, or $54 \mathrm{sec}$ during Days 1-5. Punishment is response contingent in that the response producing reinforcement also initiates the punishment delay. Camp, Raymond, and Church (1967) demonstrated an inverse relationship between punishment delay and response suppression which supports this suggestion.

However, punishment delay does not appear to be an important variable in this experiment. The common effect of generalized response suppression with contingent punishment (Camp et al., 1967, Experiment 2) was minimal. Group 1 suppressed on nonlight sequences, but suppression was limited to the early segments of the FI. The tendency of Group 2 to suppress during the $12 \mathrm{sec}$ immediately following the aversive light and the double scallop response pattern in Group 3 (see lower panel of Figure 2) could be interpreted as limited generalized suppression effects. However, the response patterns of Groups 2 and 3 could also be attributed to an $S^{\Delta}$ function for the light. Azrin (1956) demonstrated that FI punishment scheduling during a variableinterval food-reinforcement schedule produces a "reverse FI scallop," with response rate decreasing as punishment approaches. While the effect of such a response pattern upon the present FI is not clear, some disruption of responding should be expected but did not occur.

The present study differs from common punishment procedures in that punishment, and the associated delay, are entirely contingent upon $50 \%$ of the reinforcement-producing responses. The more common procedure of punishing each response (Azrin 
\& Holz, 1961; Camp et al., 1967, Experiment 4) or combining punishment with a variable-interval schedule (Camp et al., Experiments 1 and 2), so that nonreinforcement-producing responses are punished, produces generalized response suppression, which was not found in this study. Apparently, either punishment is not a viable explanation or the temporal cues and the punishment contingency exclusively with reinforcement-producing responses alters normal punishment suppression effects.

The conditioned reinforcement concept is an alternative explanation that could account for these findings. Conditioned reinforcer properties would minimize response suppression when the stimulus is paired with reinforcement and reduce response suppression during transfer. However, explanation of response facilitation following the stimulus in transfer would then require a discriminative stimulus function for light offset. While this is possible, it does not seem parsimonious in comparison to the use of a discriminative stimulus interpretation alone. The present data do not provide a clear rejection of either interpretation. It does seem clear, however, that the association of an aversive stimulus with reinforcement serves to reduce the suppressive control exerted by that stimulus.

\section{REFERENCES}

AzRIN, N. H. Some effects of two intermittent schedules of immediate and non-immediate punishment. The Journal of Psychology, 1956, 42, 3-21.
Azrin, N. H., \& Holz, W. C. Punishment during fixed-interval reinforcement. Journal of the Experimental Analysis of Behavior, 1961, 4, 343-347.

Camp, D. S., Raymond, G. A., \& ChuRch, R. M. Temporal relationship between response and punishment. Journal of Experimental Psychology, 1967, 74, 114-123.

Cohen, S. L., \& StubBs, D. A. Discriminative properties of briefly presented stimuli. Journal of the Experimental Analysis of Behavior, 1976, 25, 15-25.

Farmer, J., \& SChOENFELd, W. N. Varying temporal placement of an added stimulus in a fixed interval schedule. Journal of the Experimental Analysis of Behavior, 1966, 9, 369-375.

Holz, W. C., \& AzRin, N. H. Interactions between the discriminative and aversive properties of punishment. Journal of the Experimental Analysis of Behavior, 1962, 5, 229-234.

SNaPPer, A. G., KadDen, R. M., Shmoff, E. H., \& SChoenfeld, W. N. Stimulus intrusion on fixed-interval responding in the rat: The effects of electric shock intensity, temporal location, and response contingency. Learning and Motivation, 1975, 6, 367-384.

(Received for publication August 10, 1976.) 